Research Article

\title{
Defatted Kenaf (Hibiscus cannabinus L.) Seed Meal and Its Phenolic-Saponin-Rich Extract Protect Hypercholesterolemic Rats against Oxidative Stress and Systemic Inflammation via Transcriptional Modulation of Hepatic Antioxidant Genes
}

\author{
Kim Wei Chan $\mathbb{D}^{1},{ }^{1}$ Maznah Ismail $\mathbb{D}^{1},{ }^{1}$ Norhaizan Mohd Esa $\mathbb{D}^{1,},{ }^{1,2}$ \\ Noorjahan Banu Mohamed Alitheen, ${ }^{1,3}$ Mustapha Umar Imam (D), ${ }^{4}$ Der Jiun Ooi, \\ and Nicholas M. H. Khong ${ }^{1}$ \\ ${ }^{1}$ Institute of Bioscience, Universiti Putra Malaysia, 43400 Serdang, Selangor, Malaysia \\ ${ }^{2}$ Department of Nutrition and Dietetics, Faculty of Medicine and Health Sciences, Universiti Putra Malaysia, 43400 Serdang, \\ Selangor, Malaysia \\ ${ }^{3}$ Department of Cell and Molecular Biology, Faculty of Biotechnology and Biomolecular Sciences, Universiti Putra Malaysia, 43400 \\ Serdang, Selangor, Malaysia \\ ${ }^{4}$ Department of Medical Biochemistry, Faculty of Basic Medical Sciences, College of Public Health, Usmanu Danfodio University, \\ $P M B$ 2346, Sokoto, Nigeria
}

Correspondence should be addressed to Kim Wei Chan; chankw_antioxidant@yahoo.com and Maznah Ismail; maznahis@upm.edu.my

Received 20 September 2017; Revised 21 January 2018; Accepted 28 February 2018; Published 22 April 2018

Academic Editor: Maura Palmery

Copyright (c) 2018 Kim Wei Chan et al. This is an open access article distributed under the Creative Commons Attribution License, which permits unrestricted use, distribution, and reproduction in any medium, provided the original work is properly cited.

\begin{abstract}
The present study aimed to investigate the antioxidant and anti-inflammatory properties of defatted kenaf seed meal (DKSM) and its phenolic-saponin-rich extract (PSRE) in hypercholesterolemic rats. Hypercholesterolemia was induced using atherogenic diet feeding, and dietary interventions were conducted by incorporating DKSM (15\% and 30\%) or PSRE (at $2.3 \%$ and $4.6 \%$, resp., equivalent to the total content of DKSM-phenolics and saponins in the DKSM groups) into the atherogenic diets. After ten weeks of intervention, serum total antioxidant capacities of hypercholesterolemic rats were significantly enhanced by DKSM and PSRE supplementation $(p<0.05)$. Similarly, DKSM and PSRE supplementation upregulated the hepatic mRNA expression of antioxidant genes (Nrf2, Sod1, Sod2, Gsr, and Gpx1) of hypercholesterolemic rats $(p<0.05)$, except for Gpx1 in the DKSM groups. The levels of circulating oxidized LDL and proinflammatory biomarkers were also markedly suppressed by DKSM and PSRE supplementation $(p<0.05)$. In aggregate, DKSM and PSRE attenuated the hypercholesterolemia-associated oxidative stress and systemic inflammation in rats, potentially by enhancement of hepatic endogenous antioxidant defense via activation of the Nrf2-ARE pathway, which may be contributed by the rich content of phenolics and saponins in DKSM and PSRE. Hence, DKSM and PSRE are prospective functional food ingredients for the potential mitigation of atherogenic risks in hypercholesterolemic individuals.
\end{abstract}

\section{Introduction}

Cardiovascular diseases (CVDs) remain as the leading cause of global mortality for the past 15 years. In 2015, CVDs had claimed 17.7 million lives, accounting for approximately $45 \%$ of all noncommunicable diseases deaths and $31 \%$ of all deaths globally [1]. Atherosclerosis is the core pathological element that underlies CVDs, contributing to over $80 \%$ of CVD-related fatalities worldwide [2]; while hypercholesterolemia is one of the most prominent risk factors for developing atherosclerosis [3]. Although hypercholesterolemia is related to excessively elevated levels of circulating total and 
non-high-density lipoprotein cholesterols in the blood, it is not solely a metabolic disorder of cholesterol homeostasis. Instead, hypercholesterolemia is indispensably associated with exacerbation of oxidative stress and inflammation, which culminates in the impairment of vascular reactivity and progression of atherogenesis [4]. The hypercholesterolemic environment activates major oxidant-producing enzymes including xanthine oxidase, NADPH oxidases (NOX), and myeloperoxidase, resulting in the excessive generation of reactive oxygen species (ROS) and oxidative stress consequently. Oxidative stress abrogates endothelial nitric oxide (NO) availability, uncouples endothelial nitric oxide synthase (eNOS), and enhances the oxidation of entrapped low-density lipoprotein (LDL) within the subendothelial space, thereby eliciting the vascular inflammation response by recruiting monocytes into the tunica intima. The unregulated uptake of oxidized LDL (oxLDL) by differentiated monocytes (macrophages) leads to the formation of foam cells, producing numerous proinflammatory and oxidative stress markers, cytokines, and growth factors, which further aggravate the atherogenic process [4-7].

Improvements in awareness of CVDs, their risk factors, and preventive behaviors have been evident around the world. The appeal in the relationship between diet and health coupled with consumer acceptance for the concept of functional foods, and better understanding of its determinants, has stimulated exponential growth of the global functional food market recently. According to the latest market report, the global cardiovascular health market was valued at USD 8.2 billion in 2016 [8]. Due to the strong correlation between hypercholesterolemia, oxidative stress, and inflammation in the pathogenesis of atherosclerosis, the search for cardioprotective functional food ingredients that possess strong antioxidant and anti-inflammatory properties in addition to cholesterol-lowering effects is receiving increasing attention from related authorities, researchers, manufacturers, and consumers [9-13].

Kenaf (Hibiscus cannabinus L.) is a commercial fibre crop, cultivated mainly for its stem and stalk for the production of biocomposites, paper, fibre boards and bioplastics, and biofuel. Kenaf seed is one of the major by-products from the kenaf plantation, and its seed oil has been extensively studied for its potential as functional edible oil [14-17]. Defatted kenaf seed meal (DKSM) is the secondary waste product yielded from the kenaf seed oil extraction process, which accounts for over $75 \%$ of its seed mass. Recently, DKSM has been increasingly proven and attested to be a novel functional edible flour with highly nutritive, antioxidative, antihypercholesterolemic, and anticancer properties. Furthermore, our findings also showed that phenolics and saponins are the two major bioactives in DKSM that correspond to the aforementioned nutraceutical properties [18$22]$. Aside from the previous reports on antioxidant properties of DKSM and its bioactive-rich extract based on chemical assays, their antioxidant effects under physiological or pathological conditions have not been delved into. Furthermore, studies of anti-inflammatory properties of DKSM and PSRE in a hypercholesterolemic in vivo model have not been reported hitherto. Hence, the objectives of the present study were to investigate the antioxidant and anti-inflammatory properties of DKSM and PSRE supplementation via a hypercholesterolemic rat model. In addition, their modulatory effects on the hepatic mRNA level of antioxidant genes were also studied. PSRE was prepared and tested along with DKSM at the equivalent levels of total DKSM-phenolics and saponins in order to determine the possible contributing roles of both bioactives in the in vivo antioxidant and antiinflammatory properties of DKSM. To date, this is the first study to report on the antioxidant and anti-inflammatory properties of DKSM and PSRE supplementation in a hypercholesterolemic rat model.

\section{Materials and Methods}

2.1. Materials. Ingredients of rat diets, that is, standard rat chow, cholesterol, cholic acid, palm oil, corn starch, full cream milk powder, and eggs, were purchased from Specialty Feeds (Glen Forrest, Australia), Amresco (Solon, OH, USA), Santa Cruz Biotechnology Inc. (Dallas, TX, USA), Yee Lee Edible Oil Sdn. Bhd. (Perak, Malaysia), Thye Huat Chan Sdn. Bhd. (Penang, Malaysia), Eaga Exports Pty Ltd. (South Perth, Australia), and Lay Hong Berhad (Klang, Selangor, Malaysia), respectively. Simvastatin was purchased from Pfizer (New York, NY, USA), while potassium persulfate, 6-hydroxy-2,5,7,8-tetramethylchroman-2-carboxylic acid (Trolox), and 2,2' -azino-bis(3-ethylbenzthiazoline-6-sulphonic acid) (ABTS) were purchased from Sigma-Aldrich Co. (St. Louis, MO, USA). All solvents of analytical grade were purchased from Merck (Darmstadt, Germany). Fixative solution (RCL2 ${ }^{\circledR}$ ) was purchased from Alphelys (Plaisir, France). Rat oxidized low-density lipoprotein (oxLDL) and interleukin 6 (IL-6) ELISA kits were purchased from Cusabio (Wuhan, Hubei, China), while rat tumour necrosis factor-alpha (TNF- $\alpha$ ) and C-reactive protein (CRP) ELISA kits were purchased from EMD Millipore, Merck (Darmstadt, Germany). GenomeLab $^{\text {тм }}$ GeXP Start Kit and RNA isolation kit (GF-TR-100 RNA Isolation Kit) were purchased from Beckman Coulter Inc. (Brea, CA, USA) and Vivantis (Selangor, Malaysia), respectively. Magnesium chloride $\left(\mathrm{MgCl}_{2}\right)$ and DNA Taq polymerase were purchased from Thermo Fisher Scientific (Pittsburgh, PA, USA).

2.2. Preparation of DKSM and PSRE. Kenaf seeds (variety V 36) were obtained from the Malaysian Kenaf and Tobacco Board in Pasir Putih, Kelantan, Malaysia, and DKSM was produced following the defatting procedures of our previous study [19]. Briefly, ground kenaf seeds were homogenized at 9500 rpm (Ultra-turrax T25 basic, IKA ${ }^{\circledR}$-WERKE GmbH \& Co. KG, Staufen, Germany) with n-hexane at the ratio of $1: 2(w: v)$ for $15 \mathrm{~min}$. Then, the mixture was filtered through Whatman number 2 filter paper. The residue (DKSM) was reextracted twice accordingly and dried in an oven at $50^{\circ} \mathrm{C}$ for $3 \mathrm{~h}$ to remove residual solvent. Finally, DKSM was passed through a 30 -mesh sieve and kept in $-20^{\circ} \mathrm{C}$ for further use. Proximate analysis showed that DKSM contained 57.09\% carbohydrate, $26.19 \%$ protein, $9.34 \%$ moisture, $6.65 \%$ ash, and $0.73 \%$ fat [19]. Besides, DKSM also contained $16.95 \%$ crude fibre. 
TABLE 1: Composition (g/100 g diet) and energy distribution of diets.

\begin{tabular}{|c|c|c|c|c|c|c|}
\hline & \multicolumn{6}{|c|}{ Rat Groups } \\
\hline & NC & $\mathrm{AD} /$ Statin & DKSM-Low & DKSM-High & PSRE-Low & PSRE-High \\
\hline \multicolumn{7}{|l|}{ Ingredient } \\
\hline Ground standard rat chow & 100.0 & 60.0 & 45.0 & 30.0 & 57.7 & 55.4 \\
\hline DKSM & & & 15.0 & 30.0 & & \\
\hline PSRE & & & & & $2.3^{*}$ & $4.6^{\#}$ \\
\hline Palm oil & & 20.0 & 20.0 & 20.0 & 20.0 & 20.0 \\
\hline Full cream milk powder & & 15.0 & 15.0 & 15.0 & 15.0 & 15.0 \\
\hline Egg yolk & & 1.5 & 1.5 & 1.5 & 1.5 & 1.5 \\
\hline Cholesterol & & 2.0 & 2.0 & 2.0 & 2.0 & 2.0 \\
\hline Cholic acid & & 0.4 & 0.4 & 0.4 & 0.4 & 0.4 \\
\hline Starch & & 1.1 & 1.1 & 1.1 & 1.1 & 1.1 \\
\hline Total & 100.0 & 100.0 & 100.0 & 100.0 & 100.0 & 100.0 \\
\hline \multicolumn{7}{|l|}{ Energy distribution } \\
\hline Protein (\% kcal) & 22.2 & 13.2 & 14.1 & 15.0 & 12.9 & 12.5 \\
\hline Carbohydrate (\% kcal) & 65.8 & 35.7 & 35.6 & 35.5 & 36.0 & 36.4 \\
\hline Fat $(\% \mathrm{kcal})$ & 12.0 & 51.1 & 50.3 & 49.5 & 51.1 & 51.1 \\
\hline Total caloric value (kcal/100 g diet) & 360.8 & 479.3 & 476.1 & 473.0 & 478.2 & 477.0 \\
\hline
\end{tabular}

*Based on $15.36 \%(w / w)$ extraction yield, which corresponds to the equivalent level of total DKSM-phenolics and saponins in the "DKSM-Low" group; ${ }^{*}$ based on $15.36 \%(w / w)$ extraction yield, which corresponds to the equivalent level of total DKSM-phenolics and saponins in the "DKSM-High" group.

Phenolic-saponin-rich extract (PSRE) containing the total phenolics and saponins of DKSM was prepared according to the extraction procedures in our previous work [18]. In brief, DKSM was refluxed in $50 \%$ aqueous ethanol for $3 \mathrm{~h}$ in the ratio of $1: 15(w: v)$. Then, the mixture was filtered through Whatman filter paper number 2. Finally, solvents in the filtrate were evaporated under reduced pressure (Rotavapor R210, Buchi, Flawil, Switzerland) followed by lyophilization (VirTis BenchTop K Freeze Dryer, SP Industries, Warminster, PA, USA) to obtain PSRE. In order to estimate the recoveries of DKSM-phenolics and saponins in PSRE, DKSM residue obtained from the aforementioned procedure was extracted with methanol under sonication for $1 \mathrm{~h}$. Then, the mixture was centrifuged at $7500 \mathrm{rpm}$ for $10 \mathrm{~min}$ at $25^{\circ} \mathrm{C}$. Subsequently, the supernatant was subjected to determination of total phenolic and saponin contents, respectively, by Folin-Ciocalteu reagent and vanillin-sulphuric acid assays $[18,23,24]$. The recoveries of phenolics and saponins in PSRE from DKSM were estimated at $97.2 \pm 0.1 \%$ and 92.5 $\pm 1.8 \%$, respectively.

Characterization of targeted bioactives in PSRE, that is, phenolics and saponins, was reported in our previous work [18], and the same batch of DKSM and PSRE was used in the present study. From our study [18], total phenolic content of PSRE was estimated at $34.44 \mathrm{mg} / \mathrm{g}$ sample, with $p$ coumaric acid $(27.72 \mathrm{mg} / \mathrm{g}$ sample), caffeic acid $(5.75 \mathrm{mg} / \mathrm{g}$ sample), (+)-catechin $(0.86 \mathrm{mg} / \mathrm{g}$ sample), and gallic acid $(0.11 \mathrm{mg} / \mathrm{g}$ sample) detected as the major phenolics present. Besides, PSRE was found to contain total and steroidal saponins of 128.66 and $0.83 \mathrm{mg}$ diosgenin equivalents/g sample, respectively. Correspondingly, DKSM contains about $5.29 \mathrm{mg} / \mathrm{g}$ sample of total phenolics, which was composed of $4.26 \mathrm{mg}$ of $p$-coumaric acid, $0.88 \mathrm{mg}$ of caffeic acid, $0.13 \mathrm{mg}$ of $(+)$-catechin, and $0.02 \mathrm{mg}$ of gallic acid. Total saponin and steroidal saponin contents of DKSM were estimated at 19.76 and $0.13 \mathrm{mg}$ diosgenin equivalents/g sample, respectively.

2.3. Animal Study. Approval for the animal study was granted by the Institutional Animal Care and Use Committee (IACUC) of Universiti Putra Malaysia (Animal Ethics Approval Number: UPM/IACUC/AUP-R065/2013). The study was conducted in accordance with the guidelines for the use of animals. Forty-two male Sprague-Dawley rats (6 weeks old, $130-150 \mathrm{~g}$ ) were housed in individual plastic cages under the controlled condition of $12 / 12 \mathrm{~h}$ light/dark cycle, at 25 to $30^{\circ} \mathrm{C}$. During the acclimatization period (1 week), all rats were fed with standard rat chow (ad libitum) and given free access to water. After that, the rats were randomly assigned into 7 different groups, each consisting of 6 rats, that is, NC: rats were fed with standard rat chow; AD: rats were fed with an atherogenic diet containing 20\% palm oil, $2 \%$ cholesterol, and $0.4 \%$ cholic acid; DKSM-Low and DKSMHigh: rats were fed with a similar diet to the $\mathrm{AD}$ group except for the replacement of rat chow with DKSM (15\% and 30\% of total diet, resp.); PSRE-Low and PSRE-High: rats were fed with a similar diet to the AD group except for the replacement of rat chow with PSRE respectively at the level of $2.3 \%$ and $4.6 \%$, of the total diet (based on $15.36 \%$ extraction yield from DKSM, which are corresponded to the equivalent levels of total DKSM-phenolics and saponins in the DKSM groups); and Statin: rats were fed with an atherogenic diet and administrated with simvastatin $(10 \mathrm{mg} / \mathrm{kg}$ body weight/ day) through oral gavage. All diet compositions and caloric values are depicted in Table 1. As shown in Table 1, replacement of DKSM and PSRE with rat chow did not significantly alter the energy distribution of atherogenic diets. Food was given based on daily isocaloric value of $30 \mathrm{kcal} / 100 \mathrm{~g}$ body 
TABLE 2: Gene name, accession number, and primer sequences used in GeXP multiplex analysis.

\begin{tabular}{|c|c|c|c|}
\hline \multirow{2}{*}{ Gene name } & \multirow{2}{*}{ Accession number } & \multicolumn{2}{|c|}{ Primer sequence (with universal tag) } \\
\hline & & Forward & Reverse \\
\hline Nrf2/Nfe2l2 & NM_031789.2 & $\begin{array}{c}\text { AGGTGACACTATAGAATATCAGT } \\
\text { TACAACTGGATGAAG }\end{array}$ & $\begin{array}{c}\text { GTACGACTCACTATAGGGAGACT } \\
\text { CATGGTCATCTACAAAT }\end{array}$ \\
\hline Sod1 & NM_017050 & $\begin{array}{c}\text { AGGTGACACTATAGAATAATATG } \\
\text { GGGACAATACACAA }\end{array}$ & $\begin{array}{c}\text { GTACGACTCACTATAGGGATCCA } \\
\text { ACATGCCTCTCT }\end{array}$ \\
\hline Sod2 & NM_017051 & $\begin{array}{c}\text { AGGTGACACTATAGAATACAGGT } \\
\text { TGCTCTTCAGC }\end{array}$ & $\begin{array}{c}\text { GTACGACTCACTATAGGGAAACT } \\
\text { CTCCTTTGGGTTCT }\end{array}$ \\
\hline Gsr & NM_053906.2 & $\begin{array}{c}\text { AGGTGACACTATAGAATAAATAA } \\
\text { ACTGGGGATTCAGAC }\end{array}$ & $\begin{array}{l}\text { GTACGACTCACTATAGGGAAGTA } \\
\text { GATTTTCACATTGTCTTTG }\end{array}$ \\
\hline Gpx1 & NM_030826 & $\begin{array}{c}\text { AGGTGACACTATAGAATATTGAG } \\
\text { AAGTTCCTGGTAGGT }\end{array}$ & $\begin{array}{c}\text { GTACGACTCACTATAGGGATTTT } \\
\text { CTGGAAATCAGGTGT }\end{array}$ \\
\hline $\mathrm{B} 2 \mathrm{~m}^{\mathrm{a}}$ & NM_012512 & $\begin{array}{c}\text { AGGTGACACTATAGAATAATGCT } \\
\text { TGCAGAGTTAAACA }\end{array}$ & $\begin{array}{c}\text { GTACGACTCACTATAGGGATGCA } \\
\text { TAAAATATTTAAGGTAAGA }\end{array}$ \\
\hline $\operatorname{Kan}(r)^{c}$ & & $\begin{array}{l}\text { GGTGACACTATAGAATAATCATC } \\
\text { AGCATTGCATTCGATTCCTGTTTG }\end{array}$ & $\begin{array}{c}\text { GTACGACTCACTATAGGGAATTC } \\
\text { CGACTCGTCCAACATC }\end{array}$ \\
\hline $\operatorname{Hprt1}^{\mathrm{a}, \mathrm{b}}$ & NM_012583 & $\begin{array}{c}\text { AGGTGACACTATAGAATATCCTC } \\
\text { ATGGACTGATTATG }\end{array}$ & $\begin{array}{c}\text { GTACGACTCACTATAGGGACTGG } \\
\text { TCATTACAGTAGCTCTT }\end{array}$ \\
\hline $\mathrm{Rpl}_{13 \mathrm{a}^{\mathrm{a}}}$ & NM_173340 & $\begin{array}{c}\text { AGGTGACACTATAGAATAATGGG } \\
\text { ATCCCTCCAC }\end{array}$ & $\begin{array}{c}\text { GTACGACTCACTATAGGGAATTT } \\
\text { TCTTCTCCACATTCTT }\end{array}$ \\
\hline
\end{tabular}

${ }^{a}$ Housekeeping genes; ${ }^{b}$ normalization gene; ${ }^{c}$ internal control supplied by Beckman Coulter Inc. (Brea, CA, USA).

weight for 10 weeks, and prefiltered tap water was supplied in water-dispensing bottles ad libitum. After 10 weeks of dietary intervention, all rats were euthanized (exsanguination by cardiac puncture under anesthesia by ketamine $(100 \mathrm{mg} / \mathrm{kg})$ and xylazine $(10 \mathrm{mg} / \mathrm{kg}))$ after an overnight fast. Fasting sera were obtained via centrifugation of collected bloods. Rats' livers were carefully excised, cleaned, and preserved in RCL $2{ }^{\circledR}$ solution at $-80^{\circ} \mathrm{C}$.

The effects of DKSM and PSRE supplementation on the cholesterol metabolism of experimental rats from the present study have been reported [22]. Biochemical analysis on rats' sera showed that atherogenic diet feeding had successfully induced hypercholesterolemia and liver steatosis in rats, as evidenced by significant elevations in hepatosomatic index and hepatic lipid content as well as levels of circulating total and LDL cholesterol, as compared to the NC group. Dietary supplementation of DKSM (DKSM-Low and DKSM-High groups), PSRE (PSRE-Low and PSRE-High groups), and simvastatin (Statin group) exerted superior antihypercholesterolemic properties in the rats, with significant suppressions of elevated total and LDL cholesterol levels. Besides, supplementation of DKSM and PSRE significantly enhanced the high-density lipoprotein (HDL) cholesterol level of hypercholesterolemic rats. Furthermore, supplementations of DKSM, PSRE, and simvastatin had successfully improved the hepatosteatosis of hypercholesterolemic rats by the significant lowering of hepatosomatic indexes and hepatic lipid contents.

2.4. Serum Total Antioxidant Capacity. Serum total antioxidant capacity of experimental rats was assessed using a modified Trolox equivalent antioxidant capacity (TEAC) assay described by Katalinic et al. [25] and Chan et al. [18]. $\mathrm{ABTS}^{\bullet+}$ stock solution was prepared by reacting $7 \mathrm{mM}$ of
ABTS with $2.45 \mathrm{mM}$ of potassium persulfate. After $18 \mathrm{~h}$ of incubation in the dark at room temperature, the stock solution was diluted with phosphate buffer saline to the absorbance of $0.70 \pm 0.02$ at $734 \mathrm{~nm}$ (PharmaSpec UV-1700, Shimadzu, Kyoto, Japan). Subsequently, $50 \mu \mathrm{L}$ of diluted serum was reacted with $950 \mu \mathrm{L}$ of adjusted $\mathrm{ABTS}^{\bullet+}$ solution for $10 \mathrm{~min}$, and the absorbance was measured at $734 \mathrm{~nm}$ (PharmaSpec UV-1700, Shimadzu, Kyoto, Japan). Trolox was used as standard, and the serum total antioxidant capacity of experimental rats was expressed as mg Trolox equivalent antioxidant capacity (TEAC)/mL serum.

2.5. Hepatic mRNA Levels of Antioxidant Genes. The primers for the gene expression study were designed by referring to the Rattus norvegicus gene sequences from the National Center for Biotechnology Information website (http://www.ncbi .nlm.nih.gov/nucleotide/) and tagged with an 18-nucleotide universal forward and 19-nucleotide universal reverse sequence, respectively. Primers were supplied by Integrated DNA Technologies (Singapore) and reconstituted in RNAse-free water. The primer sequences of 5 antioxidant genes, 3 housekeeping genes, and an internal control (Kanr) for the rat hepatic multiplex panel are shown in Table 2.

Rat hepatic RNA was extracted using an RNA isolation kit according to the manufacturer's instructions, while the processes of reverse transcription (RT) and polymerase chain reaction (PCR) were conducted according to the Genome$\mathrm{Lab}^{\mathrm{TM}}$ GeXP Start Kit protocol. Multiplex universal reverse primers and $50 \mathrm{ng}$ extracted RNA were used for RT in an XP Thermal Cycler (BIOER Technology, Hangzhou, Zhejiang, China) under the following conditions: $48^{\circ} \mathrm{C}$ for $1 \mathrm{~min}, 37^{\circ} \mathrm{C}$ for $5 \mathrm{~min}, 42^{\circ} \mathrm{C}$ for $60 \mathrm{~min}, 95^{\circ} \mathrm{C}$ for $5 \mathrm{~min}$, and then held at $4^{\circ} \mathrm{C}$. Subsequently, the cDNA product $(9.3 \mu \mathrm{L})$ was mixed with $2 \mu \mathrm{L}$ of $200 \mathrm{nM}$ forward universal primers, 
$4 \mu \mathrm{L} 25 \mathrm{mM} \mathrm{MgCl}_{2}, 0.7 \mu \mathrm{L}$ of Thermo Start Taq DNA polymerase, and $4 \mu \mathrm{L}$ of $5 \mathrm{x}$ PCR Master Mix buffer and subjected to PCR in an XP Thermal Cycler (BIOER Technology, Hangzhou, Zhejiang, China) under the following conditions: initial denaturation at $95^{\circ} \mathrm{C}$ for $10 \mathrm{~min}$, followed by two-step cycles of $94^{\circ} \mathrm{C}$ for $30 \mathrm{~s}$ and $55^{\circ} \mathrm{C}$ for $30 \mathrm{~s}$, ending in a singleextension cycle of $68^{\circ} \mathrm{C}$ for $1 \mathrm{~min}$.

The PCR products obtained from previous steps were analyzed using GeXP GenomeLab Genetic Analysis System (Beckman Coulter Inc., Brea, CA, USA). In brief, $1 \mu \mathrm{L}$ of PCR products was mixed with $38.5 \mu \mathrm{L}$ sample loading solution and $0.5 \mu \mathrm{L}$ DNA size standard 400 (provided in the GenomeLab GeXP Start Kit) on a 96-well sample plate before loading on the machine. Results were analysed with the Fragment Analysis Module of the GeXP system software and normalized on the Express Profiler software.

2.6. Circulating Oxidized Low-Density Lipoprotein and Proinflammatory Biomarkers. Fasting sera of rats were subjected to immunoassays (ELISA kits) for determination of circulating oxidized LDL (oxLDL) and proinflammatory biomarkers according to the manufacturer's instructions. The levels of circulating oxLDL and C-reactive protein (CRP) were expressed in $\mathrm{ng} / \mathrm{mL}$ and $\mu \mathrm{g} / \mathrm{mL}$ serum, respectively, while levels of circulating tumour necrosis factor-alpha (TNF- $\alpha$ ) and interleukin 6 (IL-6) were determined as pg/ $\mathrm{mL}$ serum.

2.7. Statistical Analysis. All results are reported as mean \pm standard deviation $(n=6)$. One-way analysis of variance (ANOVA), accompanied with Tukey's multiple comparison test (GraphPad Prism 6.01, GraphPad Software Inc., La Jolla, CA, USA), was conducted to identify significant differences between samples $(p<0.05)$.

\section{Results and Discussion}

3.1. Serum Total Antioxidant Capacity. Oxidative stress is closely associated with the pathogenesis of atherosclerosis [6]. Thus, serum/plasma total antioxidant capacity (TAC) may represent a useful tool in assessing the global oxidative stress and antioxidant defense levels in experimental animals and human subjects [26-30]. Trolox equivalent antioxidant capacity (TEAC) assay is one of the most common assays employed in the assessment of serum TAC based on the spectrophotometric measurement of $\mathrm{ABTS}^{\bullet+}$ cation reduction (decay of green-blue chromophore absorbance) by serum antioxidative components, in comparison to the control antioxidant, Trolox (hydrophilic analogue of vitamin E) [31].

Figure 1 depicts serum TAC of experimental rats after 10 weeks of dietary intervention. Atherogenic diet feeding significantly lowered serum TAC of hypercholesterolemic rats in the $\mathrm{AD}$ group $(p<0.05)$. This finding is in agreement with several in vivo studies involving diet-induced hypercholesterolemic/hyperlipidemic rats $[10,32,33]$. The depletion of serum TAC was probably due to the override of in vivo antioxidant defense by excessive generation of oxidants/ROS under hypercholesterolemic condition. In contrast, simvastatin treatment significantly improved serum TAC of

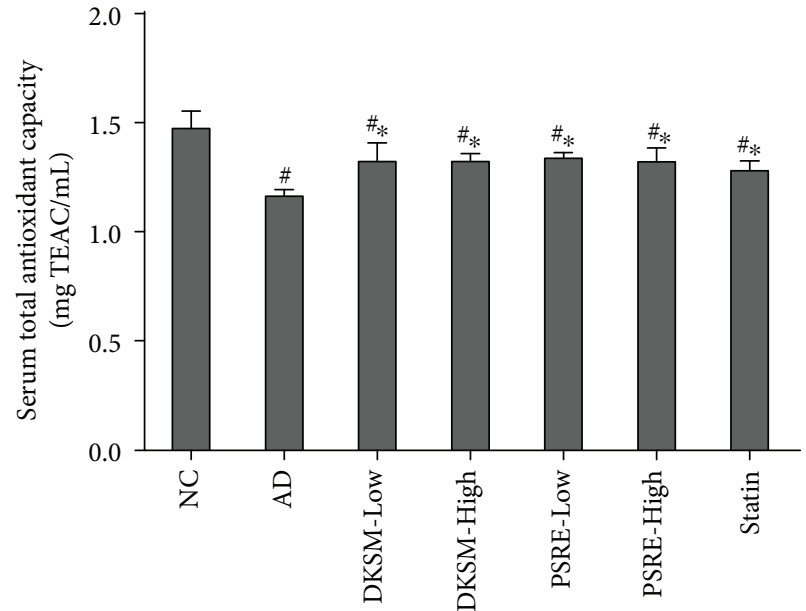

Rat Groups

FIgURE 1: Serum total antioxidant capacity after 10 weeks of dietary intervention. Data represent the mean of each group, accompanied with standard deviation. Symbols " $*$ " and "\#” respectively represent significant difference between samples in comparison to the AD and NC groups $(p<0.05)$.

hypercholesterolemic rats $(p<0.05)$, affirming the pleiotropic antioxidant properties of simvastatin [34].

As compared to the $\mathrm{AD}$ group, supplementation of DKSM and PSRE effectively counteracted the decrease in serum TAC induced by hypercholesterolemia $(p<0.05)$. The improvement in serum TAC in hypercholesterolemic rats was probably due to high antioxidant properties of DKSM and PSRE, which is supported by our previous studies using in vitro assays based on different mechanisms $[18,19]$. Consumption of an antioxidant-rich diet (e.g., fruits and vegetable which are rich in polyphenols) is strongly correlated with the improvement of antioxidant status and the attenuation of atherogenic risks in human subjects [28, 35-37].

In the present study, phenolics and saponins may have substantially contributed to the in vivo antioxidant properties of DKSM and PSRE since serum TAC between the DKSM groups and their corresponding PSRE groups (which contained the equivalent levels of DKSM-phenolics and saponins with the DKSM groups) were insignificantly different $(p>$ $0.05)$. In agreement to our previous study [18], phenolics and saponins had significantly contributed to the antioxidant properties of PSRE and DKSM. This postulation is further supported by a number of studies reporting on the in vivo antioxidant properties of major phenolic compounds detected in DKSM and PSRE, that is, $p$-coumaric acid, caffeic acid, (+)-catechin, and gallic acid. For instance, oral administration of $p$-coumaric acid and gallic acid at the dosage of $100 \mathrm{mg} / \mathrm{kg}$ body weight for 2 weeks was found to greatly improve the cardiac and hepatic total antioxidant capacities of healthy rats $[38,39]$, while dietary supplementation with caffeic and coumaric acids ( $0.2 \%$ of total diet) for 6 weeks effectively enhanced the in vivo antioxidant capacity of hypercholesterolemic rats [40]. On the other hand, plasma and urine TAC of Wistar rats was significantly increased following 10 days of intraperitoneal administration of catechin 
TABLE 3: Hepatic antioxidant gene expressions after 10 weeks of dietary intervention.

\begin{tabular}{|c|c|c|c|c|c|}
\hline \multirow{2}{*}{ Rat groups } & \multicolumn{5}{|c|}{ Hepatic antioxidant genes (relative expression) } \\
\hline & Nrf2 & Sod1 & Sod2 & Gsr & Gpx1 \\
\hline $\mathrm{NC}$ & $1.00 \pm 0.09$ & $1.00 \pm 0.11$ & $1.00 \pm 0.20$ & $1.00 \pm 0.20$ & $1.00 \pm 0.11$ \\
\hline $\mathrm{AD}$ & $0.68 \pm 0.19^{\#}$ & $0.56 \pm 0.10^{\#}$ & $0.67 \pm 0.12^{\#}$ & $0.57 \pm 0.11^{\#}$ & $0.61 \pm 0.12^{\#}$ \\
\hline DKSM-Low & $1.21 \pm 0.08^{*}$ & $0.76 \pm 0.12^{* \#}$ & $0.88 \pm 0.12$ & $0.82 \pm 0.08^{*}$ & $0.50 \pm 0.04^{\#}$ \\
\hline DKSM-High & $1.23 \pm 0.18^{* \#}$ & $0.76 \pm 0.09^{* \#}$ & $1.02 \pm 0.17^{*}$ & $0.83 \pm 0.12^{*}$ & $0.66 \pm 0.09^{\#}$ \\
\hline PSRE-Low & $1.02 \pm 0.16^{*}$ & $1.57 \pm 0.09^{* \#}$ & $2.09 \pm 0.17^{* \#}$ & $1.28 \pm 0.04^{* \#}$ & $1.71 \pm 0.32^{* \#}$ \\
\hline PSRE-High & $1.16 \pm 0.17^{*}$ & $1.53 \pm 0.11^{* \#}$ & $2.18 \pm 0.40^{* \#}$ & $1.37 \pm 0.17^{* \#}$ & $1.64 \pm 0.29^{* \#}$ \\
\hline Statin & $1.69 \pm 0.04^{* \#}$ & $1.75 \pm 0.03^{* \#}$ & $1.74 \pm 0.12^{* \#}$ & $1.42 \pm 0.23^{* \#}$ & $2.04 \pm 0.28^{* \#}$ \\
\hline
\end{tabular}

Symbol "*" within the same column indicates significant difference in comparison to the AD group $(p<0.05)$; symbol "\#” within the same column indicates significant difference in comparison to the NC group $(p<0.05)$; abbreviations: Nrf2: nuclear factor erythroid 2-related factor 2; Sod1: cytosolic superoxide dismutase; Sod2: mitochondrial superoxide dismutase; Gsr: glutathione-disulfide reductase; Gpx1: glutathione peroxidase 1.

mixture (23 mg/kg body weight) [41]. Besides phenolic compounds, saponins have also been proposed as a group of dietary phytochemicals with distinctive in vivo antioxidant properties [42, 43]. For example, supplementation with total saponins extracted from three medicinal species of Dioscorea and dry root tuber of Trichosanthis kirilowii were found to effectively improve the in vivo antioxidant capacity of myocardial ischemic rats and carbon tetrachloride-intoxicated mice, respectively $[44,45]$.

3.2. Expression of Hepatic Antioxidant Genes. Endogenous antioxidant defense plays a critical role in restoring the cellular redox imbalance caused by oxidative insults, whilst consumption of high antioxidative phytochemicals (particularly phenolic compounds) has been implicated in the enhancement of endogenous antioxidant defense via modulation of multiple redox mechanisms [46, 47]. Table 3 shows the mRNA levels of hepatic antioxidant genes (nuclear factor erythroid 2-related factor 2 (Nrf2 or Nfe2l2), cytosolic superoxide dismutase (Sod1), mitochondrial superoxide dismutase (Sod2), glutathione-disulfide reductase (Gsr), and glutathione peroxidase $1(\mathrm{Gpx} 1))$ in the experimental rats, as influenced by different dietary interventions.

After 10 weeks of atherogenic diet feeding, hepatic antioxidant gene expression (Nrf2, Sod1, Sod2, Gsr, and Gpx1) in the AD group was adversely altered $(p<0.05)$ in comparison to the $\mathrm{NC}$ group, suggesting the manifestation of hypercholesterolemia-induced oxidative stress in these rats. On the contrary, hepatic antioxidant gene expressions in the Statin group were significantly enhanced $(p<0.05)$. In consonance with previous studies, diet-induced hypercholesterolemia has been associated with the exacerbation of oxidative stress and compromised endogenous antioxidant defense in the experimental animals, while statin treatment is the effective pharmaceutical approach in reversing these deleterious impacts $[10,13,32,48,49]$.

In the present study, supplementation with DKSM and PSRE substantially improved the endogenous antioxidant defense of hypercholesterolemic rats via transcriptional modulation of hepatic antioxidant genes. In comparison to the AD group, hepatic Nrf2 gene expression of all DKSMand PSRE-supplemented rats was significantly elevated by 1.5 - to 1.8 -folds $(p<0.05)$. DKSM supplementation resulted in the upregulation of hepatic Sod1, Sod2, and Gsr expressions, especially in the DKSM-High group $(p<0.05)$. However, supplementation with DKSM did not improve the hepatic Gpxl gene expression of hypercholesterolemic rats $(p>0.05)$. Similarly, PSRE supplementation upregulated the hepatic gene expressions of Sod1, Sod2, Gsr, and Gpx1 of hypercholesterolemic rats by 2 - to 3 -folds $(p<0.05)$. Except for Nrf2, both PSRE groups exhibited superior upregulatory effects in the expressions of all studied hepatic antioxidant genes than their corresponding DKSM groups, which contained an equivalent level of total DKSMphenolics and saponins $(p<0.05)$. This is probably due to the enhancement in the release of bioactives (phenolics and saponins) from the DKSM matrix during the heated reflux extraction process, which may then result in the better bioavailability and bioefficiency of PSRE. Appropriate increase in the extraction temperature will disrupt the integrity of the cell wall, thus facilitating the release of bound bioactives from the matrix and enhancing the solubility and diffusion coefficient of bioactives into the extraction solvent, leading to the optimal recovery of bioactives in the extract $[50,51]$. For instance, heated reflux extraction $\left(80^{\circ} \mathrm{C}\right)$ of Pterodon emarginatus vogel seeds with $70 \%$ aqueous ethanol provided the highest phenolic recovery as compared to the nonthermal extraction processes [52], while a higher recovery of chickpeasaponin B1 was observed when the aqueous-ethanolic extraction processes were carried out under heated reflux condition $\left(90^{\circ} \mathrm{C}\right)$ in comparison to the nonthermal ultrasonic extraction [53].

In the present study, results from hepatic antioxidant gene expression analysis aggregately suggest that supplementation with DKSM and PSRE could have activated the hepatic Nrf2-ARE pathway in the experimental rats and consequently improved their endogenous antioxidant defense against hypercholesterolemia-induced oxidative stress. Besides, supplementation with PSRE at an equivalent level of DKSM-phenolics and saponins produced similar or superior modulatory effects on the hepatic antioxidant gene expressions than on their corresponding DKSM counterparts. This finding signifies the contributory roles of phenolics and saponins as the dietary Nrf2-ARE-activating factors in DKSM and PSRE. In recent years, activation of Nrf2ARE has been proposed as the targeted therapeutic pathway 
TABLE 4: Circulating oxidized low-density lipoprotein and proinflammatory biomarkers after 10 weeks of dietary intervention.

\begin{tabular}{lcccc}
\hline Rat groups & oxLDL $(\mathrm{ng} / \mathrm{mL})$ & TNF- $\alpha(\mathrm{pg} / \mathrm{mL})$ & IL-6 $(\mathrm{pg} / \mathrm{mL})$ & $\mathrm{CRP}(\mu \mathrm{g} / \mathrm{mL})$ \\
\hline NC & $26.90 \pm 2.29$ & $20.75 \pm 3.86$ & $3.08 \pm 0.18$ & $798.42 \pm 55.95$ \\
AD & $35.90 \pm 3.71^{\#}$ & $83.00 \pm 19.08^{\#}$ & $4.22 \pm 0.20^{\#}$ & $1018.56 \pm 155.09^{\#}$ \\
DKSM-Low & $21.49 \pm 0.83^{* \#}$ & $46.75 \pm 7.72^{* \#}$ & $3.66 \pm 0.25$ & $749.31 \pm 42.60^{*}$ \\
DKSM-High & $15.35 \pm 2.85^{* \#}$ & $45.25 \pm 9.78^{* \#}$ & $2.81 \pm 0.40^{*}$ & $624.41 \pm 42.07^{* \#}$ \\
PSRE-Low & $23.79 \pm 1.72^{*}$ & $50.50 \pm 8.39^{* \#}$ & $3.23 \pm 0.30^{*}$ & $727.67 \pm 67.66^{*}$ \\
PSRE-High & $16.81 \pm 1.34^{* \#}$ & $33.00 \pm 2.00^{*}$ & $3.44 \pm 0.88^{*}$ & $725.22 \pm 54.81^{*}$ \\
Statin & $20.85 \pm 5.64^{* \#}$ & $52.50 \pm 6.56^{* \#}$ & $3.92 \pm 0.68^{\#}$ & $683.39 \pm 77.96^{*}$ \\
\hline
\end{tabular}

Symbol " $*$ " within the same column indicates significant difference in comparison to the AD group $(p<0.05)$; symbol "\#” within the same column indicates significant difference in comparison to the NC group $(p<0.05)$; abbreviations: oxLDL: oxidized low-density lipoprotein; TNF- $\alpha$ : tumour necrosis factoralpha; IL-6: interleukin 6; CRP: C-reactive protein.

for a wide array of degenerative and immunological diseases, particularly CVDs, whilst a number of dietary phytochemicals especially polyphenols, isothiocyanates, organosulfur compounds, saponins, and curcumin are prominent natural activators of this pathway $[46,54-56]$. Nrf2 is a critical transcription factor that regulates the antioxidant responses against oxidative insults. Once it is activated, Nrf2 binds to the antioxidant response element (ARE) in the nucleus to upregulate a vast array of antioxidative and electrophile detoxification genes, such as Sod, Gsr, and Gpx [57]. Antioxidant enzymes are the core pillars of endogenous antioxidant defense that cohesively shield our body from oxidative damage and its related pathogenesis [58]. For instance, Sod is one of the most effective primary antioxidant enzymes that catalyses the conversion of superoxide anions to hydrogen peroxide, while Gpx renders hydrogen peroxide and other organic hydroperoxides (e.g., lipid peroxide) into inert end products. On the other hand, Gsr serves as an important secondary antioxidant enzyme that maintains the proper function of primary antioxidant enzymes (e.g., Gpx) by catalysing the reduction process of glutathione disulfide (GSSG) to glutathione (GSH) with NADPH as the reducing cofactor.

Interestingly, simvastatin treatment and PSRE supplementation did not only neutralize the deleterious effects of hypercholesterolemia on the transcriptions of hepatic antioxidant genes but their hepatic expressions of antioxidant genes were upregulated to a higher degree than those of the NC group $(p<0.05)$. In the present study, PSRE supplementation and simvastatin treatment significantly improved the severity of hypercholesterolemia in the experimental rats and thus produced a milder oxidative stress condition as compared to the AD group. Mild/moderate oxidative stress, simvastatin, and polyphenols have been previously reported as the activators of the Nrf2-ARE pathway by inducing the dissociation of Nrf2 from the Kelch-like ECH-associated protein-1 (Keap1) and consequently upregulating the expressions of its downstream antioxidant genes [55, 59, 60]. Although the hepatic Nrf2 expression between the NC and PSRE groups were indifferent $(p>0.05)$, the higher expressions of other hepatic antioxidant genes in the PSRE and Statin groups could be possibly explained by the enhancement of Nrf2-ARE activation through the combinatorial effects of improved oxidative stress condition as well as the inductions by simvastatin or PSRE bioactives. In agreement, the similar findings have been previously observed in the diet-induced hyperlipidemic rats, supplemented with phenolic-rich extract from Clinacanthus nutans and simvastatin [10].

3.3. Circulating Oxidized Low-Density Lipoprotein and Proinflammatory Biomarkers. Chronic hypercholesterolemia triggers excessive ROS generation, compromises endogenous antioxidant defense, and consequently results in the formation of oxidatively modified LDL/oxidized LDL (oxLDL) [4]. Circulating oxLDL level is one of the most important oxidative stress-related biomarkers, which is strongly correlated to the prevalence of atherosclerotic CVD [61-63]. Circulating oxLDL and proinflammatory biomarker levels of experimental rats after 10 weeks of dietary intervention are depicted in Table 4. In comparison to the NC group, atherogenic diet feeding significantly elevated the circulating oxLDL level of the $\mathrm{AD}$ group $(p<0.05)$, indicating the successful induction and advanced manifestation of hypercholesterolemia-induced oxidative stress in these rats. In contrast, dietary supplementation with DKSM and PSRE effectively lowered the circulating oxLDL level of hypercholesterolemic rats by $34 \%$ to $57 \%$, in a dose-dependent manner of DKSM-High $\geq$ PSRE-High $\geq$ Statin $\geq$ DKSM-Low $\geq$ PSRELow $>$ AD $(p<0.05)$. Remarkably, supplementation with higher concentration of DKSM (DKSM-High group) exhibited superior LDL oxidation inhibitory activity than simvastatin treatment $(p<0.05)$. Since there was no significant difference in the circulating oxLDL levels between the DKSM groups and their corresponding PSRE groups $(p>0.05)$, it is suggested that phenolics and saponins could have contributed to the antioxidant and LDL oxidation inhibitory properties of DKSM and PSRE. This deduction is further supported by our previous studies, of which the phenolic-saponin-rich fraction obtained via partial purification of DKSM ethanolic extract exhibited superior antioxidant properties than its bioactive-deficient counterpart [18]. Furthermore, some of the major phenolics in DKSM and PSRE, that is, $p$-coumaric acid, caffeic acid, and (+)-catechin, have been previously reported as promising natural inhibitors against LDL oxidation via in vitro and in vivo models [64-67]. 
Due to the critical roles of circulating TNF- $\alpha$, IL- 6 , and CRP in the pathogenesis of atherosclerosis, these proinflammatory biomarkers are frequently used as promising panel for the assessment of cardiovascular risks [68, 69]. In the present study, hypercholesterolemia had evidently induced systemic inflammation in the experimental rats fed on an atherogenic diet. In comparison to the NC group, significant elevations of circulating TNF- $\alpha$, IL-6, and CRP levels by 4-, $1.4-$, and 1.3-folds, respectively, were observed in the hypercholesterolemic rats from the $\mathrm{AD}$ group $(p<0.05)$. After 10 weeks of DKSM and PSRE supplementation, circulating TNF- $\alpha$ levels of hypercholesterolemic rats were markedly reduced by 39 to $60 \%$ in a dose-dependent order, that is, PSRE-High $\geq$ DKSM-High $\geq$ DKSM-Low $\geq$ PSRE-Low $\geq$ Statin $>\operatorname{AD}(p<0.05)$. A significantly lower circulating IL-6 level in the experimental rats was observed in the DKSMHigh (-33.4\%), PSRE-Low (-23.5\%), and PSRE-High $(-18.5 \%)$ groups in comparison to the $\mathrm{AD}$ group $(p<0.05)$, while simvastatin treatment (Statin group) and DKSM supplementation at a lower level (DKSM-Low group) produced insignificant lowering effects on the circulating IL-6 level $(p>0.05)$. Similar to the trend of the TNF- $\alpha$ level, supplementation with DKSM and PSRE effectively repressed the circulating CRP level of hypercholesterolemic rats in a dose-dependent manner, that is, DKSM-High $\geq$ Statin $\geq$ PSRE-High $\geq$ PSRE-Low $\geq$ DKSM-Low $>$ AD $(p<0.05)$. Furthermore, there were no significant differences in the levels of proinflammatory biomarkers between DKSM groups and their corresponding PSRE groups $(p>0.05)$, suggesting that phenolics and saponins might be the key bioactives that have contributed to the anti-inflammatory properties of DKSM and PSRE.

Elevated level of oxLDL is correlated with the upregulation of proinflammatory mediators (e.g., TNF- $\alpha$, IL-6, and CRP) in human subjects [62]. In the present study, the level of circulating oxLDL was strongly correlated with the level of CRP $(r=0.9390)$ and moderately correlated with the TNF- $\alpha(r=0.5682)$ and IL-6 $(r=0.5892)$ levels, thus affirming the etiological role of hypercholesterolemia-induced oxidative stress in eliciting systemic inflammation and higher atherogenic risk in the rats. Moreover, these correlations also suggest that the lower systemic inflammation observed in DKSM- and PSRE-supplemented rats may in part be due to the inhibition of LDL oxidation by these dietary interventions. Supplementation with DKSM and PSRE modulated the upregulation of hepatic antioxidant gene expressions as well as the enhancement of circulating nonenzymatic low molecular weight antioxidant levels (as evidenced by improvement in serum TAC) in the hypercholesterolemic rats. Thus, it is postulated that these antioxidative effects might have advantageously controlled hypercholesterolemia-induced ROS overproduction in the rats and therefore reduced the severity of LDL oxidative damage and systemic inflammation. Although PSRE supplementation showed superior upregulatory effects than its DKSM counterpart in the hepatic expressions of antioxidant genes, similar effects were not observed in TAC, oxLDL, and proinflammatory biomarker assays. This is possibly due to the relatively high levels of DKSM and
PSRE used in the present study, hence resulting in the optimal in vivo antioxidant and anti-inflammatory effects (i.e., plateau portion of the dose-response curve) observed under the tested physiological condition. On the other side, DKSM and PSRE supplementation might have exhibited an all-or-none effect notwithstanding the transcriptional changes in the in vivo model. This is probably due to the posttranscriptional modifications that produced therapeutic effects to the same degree irrespective of the degree of transcriptional changes induced. Investigations on dietary effects of DKSM and PSRE on endogenous antioxidant defense in hypercholesterolemic animal models at posttranscriptional and translational levels are suggested for further studies.

\section{Conclusion}

DKSM and its derived PSRE supplementation improved in vivo antioxidant defense of hypercholesterolemic rats possibly via transcriptional activation of hepatic Nrf2-ARE pathway and improvement of serum TAC. The enhancement in endogenous antioxidant defense therefore meritoriously inhibited the oxidation of LDL and systematic inflammatory response in the hypercholesterolemic rats. Phenolics and saponins are suggested as the key antioxidant and anti-inflammatory bioactives in DKSM and PSRE. Finally, DKSM and PSRE could be potentially used as cardioprotective functional food ingredients in counteracting hypercholesterolemia-associated oxidative stress and systemic inflammation.

\section{Conflicts of Interest}

The authors declare no conflict of interest.

\section{Acknowledgments}

The authors are thankful to the Institute of Bioscience, Universiti Putra Malaysia for all the constructive technical support and assistance. This project is funded by the Ministry of Science, Technology and Innovation (MOSTI), Malaysia, via eScienceFund (Project no. 02-01-04-SF1597).

\section{References}

[1] WHO, World Health Statistics 2017: Monitoring Health for the SDGs Sustainable Development Goals, World Health Organization, Geneva, Switzerland, 2017.

[2] GBD 2013 Mortality and Causes of Death Collaborators, "Global, regional, and national age-sex specific all-cause and cause-specific mortality for 240 causes of death, 1990-2013: a systematic analysis for the Global Burden of Disease Study 2013," The Lancet, vol. 385, no. 9963, pp. 117-171, 2015.

[3] D. Steinberg, "Hypercholesterolemia and inflammation in atherogenesis: two sides of the same coin," Molecular Nutrition and Food Research, vol. 49, no. 11, pp. 995-998, 2005.

[4] P. A. Stapleton, A. G. Goodwill, M. E. James, R. W. Brock, and J. C. Frisbee, "Hypercholesterolemia and microvascular dysfunction: interventional strategies," Journal of Inflammation, vol. 7, no. 1, p. 54, 2010. 
[5] D. Steinberg, "Atherogenesis in perspective: hypercholesterolemia and inflammation as partners in crime," Nature Medicine, vol. 8, no. 11, pp. 1211-1217, 2002.

[6] U. Singh and I. Jialal, "Oxidative stress and atherosclerosis," Pathophysiology, vol. 13, no. 3, pp. 129-142, 2006.

[7] M. Hulsmans and P. Holvoet, "The vicious circle between oxidative stress and inflammation in atherosclerosis," Journal of Cellular and Molecular Medicine, vol. 14, no. 1-2, pp. 70-78, 2009.

[8] Euromonitor International, Healthy Ageing: Opportunities in Cardiovascular and Bone Health Positioned Foods and Beverages, Euromonitor International, London, UK, 2017.

[9] C.-C. Lu and G.-C. Yen, "Antioxidative and anti-inflammatory activity of functional foods," Current Opinion in Food Science, vol. 2, pp. 1-8, 2015.

[10] N. Sarega, M. U. Imam, D.-J. Ooi et al., "Phenolic rich extract from Clinacanthus nutans attenuates hyperlipidemiaassociated oxidative stress in rats," Oxidative Medicine and Cellular Longevity, vol. 2016, Article ID 4137908, 16 pages, 2016.

[11] Y. J. Lee, Y. Ahn, O. Kwon et al., "Dietary wolfberry extract modifies oxidative stress by controlling the expression of inflammatory mRNAs in overweight and hypercholesterolemic subjects: a randomized, double-blind, placebocontrolled trial," Journal of Agricultural and Food Chemistry, vol. 65, no. 2, pp. 309-316, 2017.

[12] A. Orem, C. Alasalvar, B. V. Kural et al., "Cardio-protective effects of phytosterol-enriched functional black tea in mild hypercholesterolemia subjects," Journal of Functional Foods, vol. 31, pp. 311-319, 2017.

[13] M. Ismail, G. Al-Naqeep, and K. W. Chan, "Nigella sativa thymoquinone-rich fraction greatly improves plasma antioxidant capacity and expression of antioxidant genes in hypercholesterolemic rats," Free Radical Biology and Medicine, vol. 48, no. 5, pp. 664-672, 2010.

[14] S. A. Abd Ghafar, M. Ismail, L. Saiful Yazan et al., "Cytotoxic activity of kenaf seed oils from supercritical carbon dioxide fluid extraction towards human colorectal cancer (HT29) cell lines," Evidence-Based Complementary and Alternative Medicine, vol. 2013, Article ID 549705, 8 pages, 2013.

[15] K. W. Chan and M. Ismail, "Supercritical carbon dioxide fluid extraction of Hibiscus cannabinus L. seed oil: a potential solvent-free and high antioxidative edible oil," Food Chemistry, vol. 114, no. 3, pp. 970-975, 2009.

[16] L. S. Yazan, J. B. Foo, S. A. A. Ghafar, K. W. Chan, P. M. Tahir, and M. Ismail, "Effect of kenaf seed oil from different ways of extraction towards ovarian cancer cells," Food and Bioproducts Processing, vol. 89, no. 4, pp. 328-332, 2011.

[17] W.-Y. Cheng, J. M. H. Akanda, and K.-L. Nyam, "Kenaf seed oil: a potential new source of edible oil," Trends in Food Science \& Technology, vol. 52, pp. 57-65, 2016.

[18] K. W. Chan, S. Iqbal, N. M. Khong, D.-J. Ooi, and M. Ismail, "Antioxidant activity of phenolics-saponins rich fraction prepared from defatted kenaf seed meal," LWT-Food Science and Technology, vol. 56, no. 1, pp. 181-186, 2014.

[19] K. W. Chan, N. M. Khong, S. Iqbal, S. M. Mansor, and M. Ismail, "Defatted kenaf seed meal (DKSM): prospective edible flour from agricultural waste with high antioxidant activity," LWT-Food Science and Technology, vol. 53, no. 1, pp. 308-313, 2013.
[20] L. S. Yazan, N. A. Rahman, K. W. Chan, W. N. H. W. A. Ghani, Y. S. Tor, and J. B. Foo, "Phenolics-saponins rich fraction of defatted kenaf seed meal exhibits cytotoxicity towards cancer cell lines," Asian Pacific Journal of Tropical Biomedicine, vol. 6, no. 5, pp. 404-409, 2016.

[21] N. M. Yusri, K. W. Chan, S. Iqbal, and M. Ismail, "Phenolic content and antioxidant activity of Hibiscus cannabinus L. seed extracts after sequential solvent extraction," Molecules, vol. 17, no. 12, pp. 12612-12621, 2012.

[22] K. W. Chan, M. Ismail, N. Mohd Esa, M. U. Imam, D. J. Ooi, and N. M. Khong, "Dietary supplementation of defatted kenaf (Hibiscus cannabinus L.) seed meal and its phenolics-saponins rich extract effectively attenuates diet-induced hypercholesterolemia in rats," Food \& Function, vol. 9, no. 2, pp. 925-936, 2018.

[23] S. Hiai, H. Oura, and T. Nakajima, "Color reaction of some sapogenins and saponins with vanillin and sulfur1c acid," Planta Medica, vol. 29, no. 2, pp. 116-122, 1976.

[24] K. W. Chan, N. M. Khong, S. Iqbal, and M. Ismail, "Isolation and antioxidative properties of phenolics-saponins rich fraction from defatted rice bran," Journal of Cereal Science, vol. 57, no. 3, pp. 480-485, 2013.

[25] V. Katalinic, D. Modun, I. Music, and M. Boban, "Gender differences in antioxidant capacity of rat tissues determined by $2,2^{\prime}$-azinobis (3-ethylbenzothiazoline 6-sulfonate; ABTS) and ferric reducing antioxidant power (FRAP) assays," Comparative Biochemistry and Physiology Part C: Toxicology \& Pharmacology, vol. 140, no. 1, pp. 47-52, 2005.

[26] C. Kusano and B. Ferrari, "Total antioxidant capacity: a biomarker in biomedical and nutritional studies," Journal of Cell and Molecular Biology, vol. 7, no. 1, pp. 1-15, 2008.

[27] S. G. Lee, T. Wang, T. M. Vance et al., "Validation of analytical methods for plasma total antioxidant capacity by comparing with urinary 8-isoprostane level," Journal of Microbiology and Biotechnology, vol. 27, no. 2, pp. 388-394, 2017.

[28] Y. Wang, M. Yang, S.-G. Lee et al., "Plasma total antioxidant capacity is associated with dietary intake and plasma level of antioxidants in postmenopausal women," The Journal of Nutritional Biochemistry, vol. 23, no. 12, pp. 1725-1731, 2012.

[29] C. P. Rubio, J. Hernández-Ruiz, S. Martinez-Subiela, A. Tvarijonaviciute, and J. J. Ceron, "Spectrophotometric assays for total antioxidant capacity (TAC) in dog serum: an update," BMC Veterinary Research, vol. 12, no. 1, p. 166, 2016.

[30] S.-J. Lee, S.-K. Choi, and J.-S. Seo, "Grape skin improves antioxidant capacity in rats fed a high fat diet," Nutrition Research and Practice, vol. 3, no. 4, pp. 279-285, 2009.

[31] N. J. Miller, C. Rice-Evans, M. J. Davies, V. Gopinathan, and A. Milner, "A novel method for measuring antioxidant capacity and its application to monitoring the antioxidant status in premature neonates," Clinical Science, vol. 84, no. 4, pp. 407$412,1993$.

[32] Z. Yida, M. U. Imam, M. Ismail et al., "Edible bird's nest attenuates high fat diet-induced oxidative stress and inflammation via regulation of hepatic antioxidant and inflammatory genes," BMC Complementary and Alternative Medicine, vol. 15, no. 1, p. 310, 2015.

[33] N. Ismail, M. Ismail, N. H. Azmi et al., "Beneficial effects of TQRF and TQ nano- and conventional emulsions on memory deficit, lipid peroxidation, total antioxidant status, antioxidants genes expression and soluble $A \beta$ levels in high fatcholesterol diet-induced rats," Chemico-Biological Interactions, vol. 275, pp. 61-73, 2017. 
[34] A. Rohilla, S. Rohilla, A. Kumar, M. Khan, and A. Deep, "Pleiotropic effects of statins: a boulevard to cardioprotection," Arabian Journal of Chemistry, vol. 9, pp. S21-S27, 2016.

[35] K. Kim, T. M. Vance, and O. K. Chun, "Greater total antioxidant capacity from diet and supplements is associated with a less atherogenic blood profile in U.S. adults," Nutrients, vol. 8, no. 1, p. 15, 2016.

[36] J. Harasym and R. Oledzki, "Effect of fruit and vegetable antioxidants on total antioxidant capacity of blood plasma," Nutrition, vol. 30, no. 5, pp. 511-517, 2014.

[37] A. Khalil, P. Gaudreau, M. Cherki et al., “Antioxidant-rich food intakes and their association with blood total antioxidant status and vitamin $\mathrm{C}$ and $\mathrm{E}$ levels in community-dwelling seniors from the Quebec longitudinal study NuAge," Experimental Gerontology, vol. 46, no. 6, pp. 475-481, 2011.

[38] C.-T. Yeh, L.-C. Ching, and G.-C. Yen, "Inducing gene expression of cardiac antioxidant enzymes by dietary phenolic acids in rats," The Journal of Nutritional Biochemistry, vol. 20, no. 3, pp. 163-171, 2009.

[39] C.-T. Yeh and G.-C. Yen, "Induction of hepatic antioxidant enzymes by phenolic acids in rats is accompanied by increased levels of multidrug resistance-associated protein $3 \mathrm{mRNA}$ expression," The Journal of Nutrition, vol. 136, no. 1, pp. 1115,2006

[40] Y.-H. Yeh, Y.-T. Lee, H.-S. Hsieh, and D.-F. Hwang, "Dietary caffeic acid, ferulic acid and coumaric acid supplements on cholesterol metabolism and antioxidant activity in rats," Journal of Food and Drug Analysis, vol. 17, no. 2, pp. 123-132, 2009.

[41] Y. V. Simos, I. I. Verginadis, I. K. Toliopoulos et al., "Effects of catechin and epicatechin on superoxide dismutase and glutathione peroxidase activity, in vivo," Redox Report, vol. 17, no. 5, pp. 181-186, 2012.

[42] J. Sidana, B. Singh, and O. P. Sharma, "Saponins of Agave: chemistry and bioactivity," Phytochemistry, vol. 130, pp. 2246, 2016.

[43] O. O. Elekofehinti, "Saponins: anti-diabetic principles from medicinal plants-a review," Pathophysiology, vol. 22, no. 2, pp. 95-103, 2015.

[44] Y. Chen, Y. Miao, L. Huang et al., "Antioxidant activities of saponins extracted from Radix Trichosanthis: an in vivo and in vitro evaluation," BMC Complementary and Alternative Medicine, vol. 14, no. 1, p. 86, 2014.

[45] Y.-N. Tang, X.-C. He, M. Ye et al., "Cardioprotective effect of total saponins from three medicinal species of Dioscorea against isoprenaline-induced myocardial ischemia," Journal of Ethnopharmacology, vol. 175, pp. 451-455, 2015.

[46] S. Qin and D. X. Hou, "Multiple regulations of Keap1/Nrf2 system by dietary phytochemicals," Molecular Nutrition \& Food Research, vol. 60, no. 8, pp. 1731-1755, 2016.

[47] S. Upadhyay and M. Dixit, "Role of polyphenols and other phytochemicals on molecular signaling," Oxidative Medicine and Cellular Longevity, vol. 2015, Article ID 504253, 15 pages, 2015.

[48] Z. Yida, M. U. Imam, M. Ismail, N. Ismail, A. Ideris, and M. A. Abdullah, "High fat diet-induced inflammation and oxidative stress are attenuated by $\mathrm{N}$-acetylneuraminic acid in rats," Journal of Biomedical Science, vol. 22, no. 1, p. 96, 2015.

[49] D. Gao, Z. Gao, and G. Zhu, "Antioxidant effects of Lactobacillus plantarum via activation of transcription factor Nrf2," Food \& Function, vol. 4, no. 6, pp. 982-989, 2013.
[50] F. Shahidi and J. Yeo, "Insoluble-bound phenolics in food," Molecules, vol. 21, no. 9, article 1216, 2016.

[51] G. Spigno and D. M. De Faveri, "Antioxidants from grape stalks and marc: influence of extraction procedure on yield, purity and antioxidant power of the extracts," Journal of Food Engineering, vol. 78, no. 3, pp. 793-801, 2007.

[52] R. C. Dutra, M. N. Leite, and N. R. Barbosa, "Quantification of phenolic constituents and antioxidant activity of Pterodon emarginatus vogel seeds," International Journal of Molecular Sciences, vol. 9, no. 12, pp. 606-614, 2008.

[53] K. Cheng, H. Gao, R.-R. Wang et al., "Evaluation of extraction and degradation methods to obtain chickpeasaponin B1 from chickpea (Cicer arietinum L.)," Molecules, vol. 22, no. 12, p. 332, 2017.

[54] J. Fan, D. Liu, C. He, X. Li, and F. He, "Inhibiting adhesion events by Panax notoginseng saponins and ginsenoside Rb1 protecting arteries via activation of Nrf2 and suppression of p38 - VCAM-1 signal pathway," Journal of Ethnopharmacology, vol. 192, pp. 423-430, 2016.

[55] A. L. Stefanson and M. Bakovic, "Dietary regulation of Keap1/ Nrf2/ARE pathway: focus on plant-derived compounds and trace minerals," Nutrients, vol. 6, no. 12, pp. 3777-3801, 2014.

[56] D. J. Ooi, K. W. Chan, N. Ismail, M. U. Imam, and M. Ismail, "Polyphenol-rich ethyl acetate fraction of Molineria latifolia rhizome restores oxidant-antioxidant balance by possible engagement of KEAP1-NRF2 and $\mathrm{PKC} / \mathrm{NF}-\kappa \mathrm{B}$ signalling pathways," Journal of Functional Foods, vol. 42, pp. 111-121, 2018.

[57] Q. Ma, "Role of Nrf2 in oxidative stress and toxicity," Annual Review of Pharmacology and Toxicology, vol. 53, no. 1, pp. 401-426, 2013.

[58] E. Birben, U. M. Sahiner, C. Sackesen, S. Erzurum, and O. Kalayci, "Oxidative stress and antioxidant defense," World Allergy Organization Journal, vol. 5, no. 1, pp. 9-19, 2012.

[59] D. Chartoumpekis, P. G. Ziros, A. Psyrogiannis, V. Kyriazopoulou, A. G. Papavassiliou, and I. G. Habeos, "Simvastatin lowers reactive oxygen species level by Nrf2 activation via PI3K/Akt pathway," Biochemical and Biophysical Research Communications, vol. 396, no. 2, pp. 463-466, 2010.

[60] H. K. Bayele, E. S. Debnam, and K. S. Srai, "Nrf2 transcriptional derepression from Keap 1 by dietary polyphenols," Biochemical and Biophysical Research Communications, vol. 469, no. 3, pp. 521-528, 2016.

[61] P. Holvoet, A. Mertens, P. Verhamme et al., "Circulating oxidized LDL is a useful marker for identifying patients with coronary artery disease," Arteriosclerosis, Thrombosis, and Vascular Biology, vol. 21, no. 5, pp. 844-848, 2001.

[62] J. Hulthe and B. Fagerberg, "Circulating oxidized LDL is associated with subclinical atherosclerosis development and inflammatory cytokines (AIR study)," Arteriosclerosis, Thrombosis, and Vascular Biology, vol. 22, no. 7, pp. 1162-1167, 2002.

[63] H. Itabe, "Oxidized low-density lipoprotein as a biomarker of in vivo oxidative stress: from atherosclerosis to periodontitis," Journal of Clinical Biochemistry and Nutrition, vol. 51, no. 1, pp. 1-8, 2012.

[64] J.-C. Cheng, F. Dai, B. Zhou, L. Yang, and Z.-L. Liu, “Antioxidant activity of hydroxycinnamic acid derivatives in human low density lipoprotein: mechanism and structure-activity relationship," Food Chemistry, vol. 104, no. 1, pp. 132-139, 2007. 
[65] L.-Y. Zang, G. Cosma, H. Gardner, X. Shi, V. Castranova, and V. Vallyathan, "Effect of antioxidant protection by p-coumaric acid on low-density lipoprotein cholesterol oxidation," American Journal of Physiology Cell Physiology, vol. 279, no. 4, pp. C954-C960, 2000.

[66] S. B. Lotito and C. G. Fraga, "(+)-Catechin prevents human plasma oxidation," Free Radical Biology and Medicine, vol. 24, no. 3, pp. 435-441, 1998.

[67] K. Osada, M. Takahashi, S. Hoshina, M. Nakamura, S. Nakamura, and M. Sugano, "Tea catechins inhibit cholesterol oxidation accompanying oxidation of low density lipoprotein in vitro," Comparative Biochemistry and Physiology Part C: Toxicology \& Pharmacology, vol. 128, no. 2, pp. 153164, 2001.

[68] A. Battistoni, S. Rubattu, and M. Volpe, "Circulating biomarkers with preventive, diagnostic and prognostic implications in cardiovascular diseases," International Journal of Cardiology, vol. 157, no. 2, pp. 160-168, 2012.

[69] S. Giovannini, G. Onder, R. Liperoti et al., "Interleukin-6, Creactive protein, and tumor necrosis factor-alpha as predictors of mortality in frail, community-living elderly individuals," Journal of the American Geriatrics Society, vol. 59, no. 9, pp. 1679-1685, 2011. 


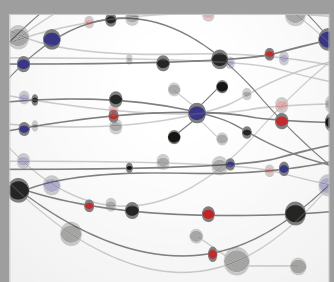

The Scientific World Journal
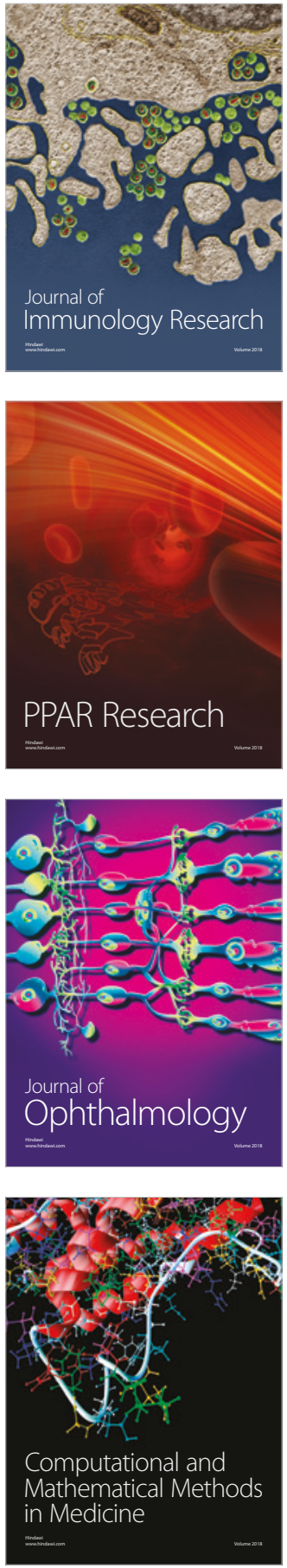

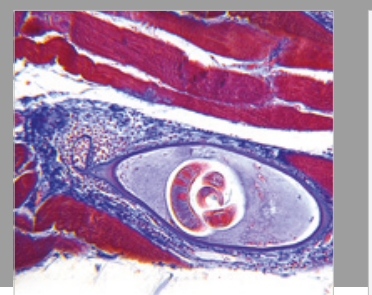

Gastroenterology Research and Practice

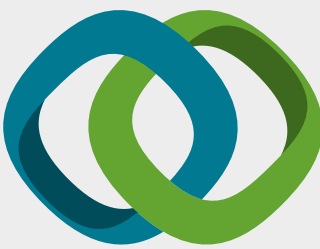

\section{Hindawi}

Submit your manuscripts at

www.hindawi.com
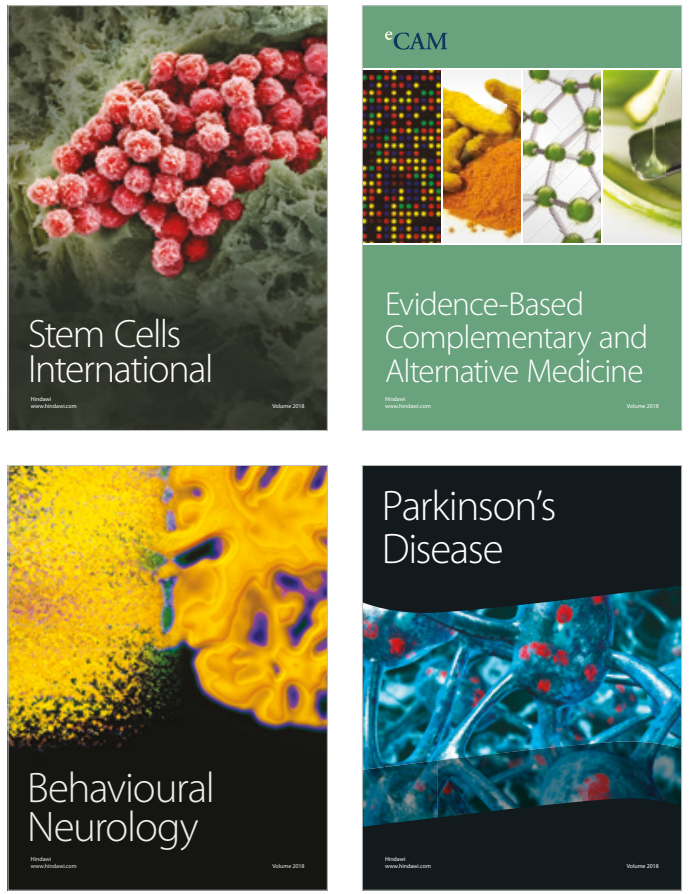

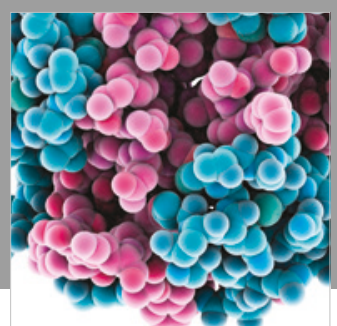

ournal of

Diabetes Research

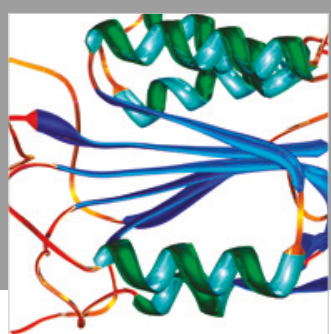

Disease Markers
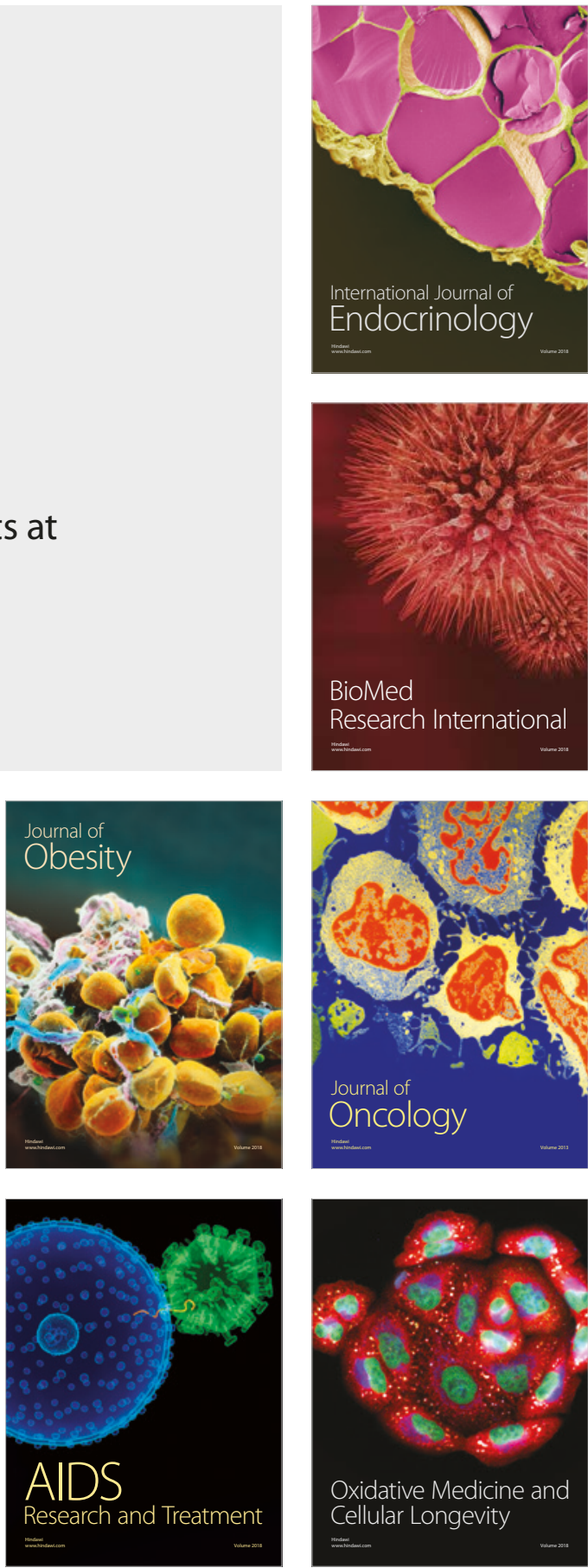